\title{
Strongyloidiasis in the COVID era: a warning for an implementation of the screening protocol
}

\author{
lacopo Vellere ${ }^{1}$. Lucia Graziani ${ }^{1} \cdot$ Marta Tilli ${ }^{1}$ - Antonia Mantella ${ }^{1} \cdot$ Irene Campolmi $^{2}$ - Jessica Mencarini ${ }^{2}$. \\ Beatrice Borchi ${ }^{2}$. Michele Spinicci ${ }^{1,2}$. Alberto Antonelli, ${ }^{1,3}$. Gian Maria Rossolini $i^{1,3}$. Alessandro Bartoloni ${ }^{1,2}$. \\ Lorenzo Zammarchi ${ }^{1,2}$ (B)
}

Received: 27 December 2020 / Accepted: 27 April 2021 / Published online: 10 May 2021

(c) Springer-Verlag GmbH Germany, part of Springer Nature 2021

\section{Dear Editor,}

We read with extreme interest the recent case report by Marchese et al. concerning the manifestation of Strongyloides stercoralis infection during immunosuppressive therapy for COVID-19 [1]. As a matter of fact, dexamethasone has clearly shown to reduce mortality for COVID-19 in a randomized clinical trial [2]. More recently, international trials showed a benefit in terms of survival with the use of IL-6 antagonists, such as tocilizumab and sarilumab [3,4]. Moreover, additional treatment options, such as higher dose of corticosteroids and other immunosuppressive drugs, are being evaluated in clinical trials, used off-label or in the context of compassionate programs [5]. The risk of reactivation of latent infections in COVID-19 patients treated with such medications may be considered limited if compared to that of patients with chronic immune-mediated diseases, since treatment course is usually shorter. However, no specific studies have addressed this point so far.

Migrants can harbor latent infections, at risk of reactivation during immunosuppressive conditions, which are widely neglected by physicians working in high-income countries, causing difficulties in screening, diagnosis and management. The guidelines from the Italian and Spanish Societies of Tropical Medicine and Rheumatology have

Iacopo Vellere, Lucia Graziani and Marta Tilli equally contributed to this article.

Lorenzo Zammarchi

lorenzo.zammarchi@unifi.it

1 Department of Experimental and Clinical Medicine, University of Florence, Florence, Italy

2 Infectious and Tropical Diseases Unit, Careggi University Hospital, Florence, Italy

3 Microbiology and Virology Unit, Careggi University Hospital, Florence, Italy identified some priority infectious diseases to be screened for in migrant candidates for treatment with immunosuppressive biological agents. In addition to HIV, HBV, HCV and latent tuberculosis infection (LTBI), taken into account in several other recommendations, the panel especially recommends screening for strongyloidiasis and Chagas disease in all patients coming from endemic areas [6]. In particular, screening for strongyloidiasis using serology and (if available) stool examination is suggested in exposed populations candidate to biological treatment, while serological screening for Chagas disease should be considered in patients coming from or whose mother was born in continental Latin America and/or in those who received blood transfusions in endemic areas. Major concerns exist about the risk of Strongyloides hyperinfection and dissemination: indeed, corticosteroids can have a hormone-like stimulating effect on larvae growth, just after a single dose [7]. Unfortunately, a case of disseminated strongyloidiasis linked to COVID-19 therapy has already been reported, indicating that this issue should be urgently considered within COVID-19 guidelines [8].

A screening protocol for infectious diseases, namely LTBI (migrants, elderly people and everyone who was treated with immunosuppressive drugs), strongyloidiasis (migrants from endemic countries and Italian patients older than 65), HBV infection (all subjects) and Chagas disease (migrants from Latin America), in COVID-19 patients potentially candidate to immunosuppressant treatments, was implemented in Careggi University Hospital, Florence, Italy. Screening for LTBI was based on an interferon gamma release assay, while for the remaining infections was based on specific serologycal tests. If possible, subjects at risk for strogyloidiasis were also tested with agar plate stool culture. Overall, one hundred hospitalized COVID-19 patients were screened for latent infections between March and August, 2020. Median age was 67.5 year (IQR 56-78), male to female ratio was 1.4:1. Six patients were migrants from Latin America $(n=5)$ 
or Central Asia $(n=1)$, while the remaining were Italians $(n=94)$. Fifty-eight patients were treated with either high dose steroids (6-methylprednisolone $>=1 \mathrm{mg} / \mathrm{Kg})(n=26)$ or immune-modulators, like tocilizumab $(n=37)$, anakinra $(n=2)$ or ruxolitinib $(n=14)$; two of them were treated with both tocilizumab and ruxolitinib. Prevalence of occult HBV infection (OBI), LTBI and strongyloidiasis were 16\% (16 out of 100$), 17 \%$ (16 out of 93 ), and 1.8\% (1 out of 54) respectively. Screening for Strongyloides was performed with both serology and agar plate stool colture in 13 patients, while the remaining 41 subjects were screened with serology only since the collection of a stool sample was not possible due to organizative issues and/or scarce patient compliance. The only patient seropositive for Strongyloides (index of 1.58, cut off 1.1) was a 75 year old man from Tuscany. His agar plate stool culture was negative (probable strongyloidiasis case). All Latin American subjects were from Peru and tested negative for Chagas disease. All patients with LTBI or OBI were reassessed after three months. There have been no cases of TB or HBV reactivation, as demonstrated by undetectable HBV viremia, and absence of constitutional symptoms or radiological imaging suggestive for TB. The patient with probable strongyloidiasis was treated with a single dose of ivermectin $200 \mathrm{mcg} / \mathrm{kg}$.

The screening strategy for strongyloidiasis among patients hospitalized for COVID-19 is challenging, and the case described by Marchese et al. [1] is a good example. The patient was a 59-year-old Italian-born subject from a Southern region, typically believed to be at low risk for Strongyloides: prevalence of strongyloidiasis in Italy is unknown, however at least Northern Regions were notoriously endemic in the past. A recent report from these regions underlines its presence in elderly people (born before 1952), with an $8 \%$ prevalence among those with eosinophilia and $1 \%$ among those with a normal eosinophils count [9]. Regarding migrants, a systematic review showed a strongyloidiasis seroprevalence of $12.2 \%$ in non-endemic countries, with the highest rate in subjects coming from East Asia and the Pacific (17.3\%), Sub-Saharan Africa (14.6\%) and Latin America and the Caribbean (11.6\%) [10]. According to another study performed in Italy, a strongyloidiasis seroprevalence of 2.5 and $1.3 \%$ was detected in migrants coming from Eastern Europe and North Africa, respectively [11]. Guidelines for screening and management of strongyloidiasis in non-endemic countries suggest that all candidates to immunosuppressive treatments at high or intermediate risk of exposure to Strongyloides, should be screened with both direct (agar plate stool culture or Baermann technique) and indirect methods (serology); in case of lack of adequate diagnostic facilities, empirical treatment with a single dose of ivermectin for one or two days is recommended [12].
Therefore, in hospitalized patients with COVID-19 a first screening strategy for strongyloidiasis could consist of testing migrants, patients exposed in tropical and subtropical regions and patients aged over 65 from areas where the disease was endemic in the past, such as the Mediterranean basin. The screening should be performed with both serology and direct examination of stools using a more sensitive technique (Baermann, agar plate stool culture or even Polymerase Chain Reaction, PCR). However, this strategy has some limitations: tests are time and resource consuming (agar plate stool culture takes at least $48 \mathrm{~h}$ to turn out positive) and serology could be falsely negative in immunocompromised subjects. Moreover, stool sample could be difficult to collect, and larvae are identified only by experienced parasitologists.

An alternative to the "test and treat" strategy could be based on the presumptive treatment of the target population with a single dose of ivermectin for one day, regardless of laboratory results, as recently suggested by Stauffer et al. [13]. This approach is also suggested by guidelines for management of solid-organ transplant recipients and donors in Latin America [14]: in hyperendemic regions where ivermectin is easily accessible, empiric treatment of all candidates (and donors) before transplant is suggested, without screening because of the risk of false negative testing. The guidelines advocate that optimal strategy depends on local epidemiology, laboratory capabilities, and drug availability.

This approach could be more pragmatic, but may present logistic drawbacks depending on the setting:in several countries, like Italy, ivermectin is unregistered and should be imported from abroad resulting in uncertain drug supplying, especially in peripheral centers [15] . Legislation in Italy requires a signed informed consent to dispense foreign drugs, and this may cause an additional barrier in elderly people with cognitive impairment or non-Italian speaking migrants; finally, Loa loa infection should be ruled out in patients from endemic regions of Sub-Saharan Africa, before administration of ivermectin to prevent possible severe encephalopathy.

Given these considerations, a hybrid approach may be proposed, tailored on non-endemic setting, like Italy in order to mitigate the risk of Strongyloides hyperinfection or dissemination in patients hospitalized for COVID-19: (i) perform serology and, if possible, agar plate stool culture (or Baerman or PCR) on intermediate-risk subjects, such as autochthonous patients above 65 years old; (ii) consider presumptive treatment in high-risk groups, such as migrants from highly endemic regions and elderly Italian patients with eosinophil count superior to $500 / \mathrm{mcL}$.

Even this approach require access to serological assay, now available only in a few tertiary or teaching hospitals, to be spread out across peripheral health centers. Indeed, 
serological screening for Strongyloides in newly arrived migrants has already been recommended by national guidelines, without implementation at a regional level [16] , probably due to budget limitations and low awareness about life-threatening consequences of the disease. Hopefully, as already mentioned by Tilli $\mathrm{M}$ et al., COVID-19 pandemic could become an opportunity to resume and strengthen screening policies about strongyloidiasis and other neglected tropical diseases in non endemic countries [17].

Funding This article has been supported by funds of the 'Ministry of Education, University and Research (Italy) Excellence Departments 2018-2022' Project for the Department of Experimental and Clinical Medicine, University of Florence, Florence, Italy.

\section{Declarations}

Conflict of interest The authors have no relevant disclosures for this article.

\section{References}

1. Marchese V, et al. Strongyloides infection manifested during immunosuppressive therapy for SARS-CoV-2 pneumonia. Infection. 2020. https://doi.org/10.1007/s15010-020-01522-4.

2. The Recovery Collaborative Group. Dexamethasone in hospitalized patients with Covid-19-preliminary report. N Engl J Med. 2020;55455:5-6. https://doi.org/10.1056/nejmoa2021436.

3. The REMAP-CAP Investigators. Interleukin-6 receptor antagonists in critically ill patients with Covid-19-preliminary report. N Engl J Med. 2021. https://doi.org/10.1056/NEJMoa2100433.

4. RECOVERY Collaborative Group. Tocilizumab in patients admitted to hospital with COVID-19 (RECOVERY): a randomised, controlled, open-label, platform trial. Lancet. 2021 May 1;397(10285):1637-1645. PMID: 33933206; PMCID: PMC8084355. https://doi.org/10.1016/S0140-6736(21)00676-0

5. Jamilloux Y, et al. Should we stimulate or suppress immune responses in COVID-19? Cytokine and anti-cytokine interventions. Autoimmun Rev. 2020;19:102567. https://doi.org/10.1016/j. autrev.2020.102567.

6. Bartalesi $\mathrm{F}$, et al. Recommendations for infectious disease screening in migrants to Western Europe with inflammatory arthropathies before starting biologic agents. Results from a multidisciplinary task force of four European societies (SIR, SER, SIMET, SEMTSI) facing the lar. Clin Exp Rheumatol. 2017;35:752-65.

7. Thomas MC, Costello SA. Disseminated strongyloidiasis arising from a single dose of dexamethasone before stereotactic radiosurgery. Int J Clin Pract. 1998;52:520-1.

8. Lier AJ, et al. Case report: disseminated strongyloidiasis in a patient with COVID-19. Am J Trop Med Hyg. 2020;103:1590-2. https://doi.org/10.4269/ajtmh.20-0699.

9. Buonfrate D, et al. Epidemiology of Strongyloides stercoralis in northern Italy: Results of a multicentre case-control study, February 2013 to July 2014. Eurosurveillance. 2016;21:1-8. https://doi. org/10.2807/1560-7917.ES.2016.21.31.30310.

10. Asundi A, et al. Prevalence of strongyloidiasis and schistosomiasis among migrants: a systematic review and meta-analysis. Lancet Glob Heal. 2019;7:e236-48. https://doi.org/10.1016/S2214109X(18)30490-X.

11. Martelli G, et al. Seroprevalence of five neglected parasitic diseases among immigrants accessing five infectious and tropical diseases units in Italy: a cross-sectional study. Clin Microbiol Infect. 2017;23:335.e1-e5. https://doi.org/10.1016/j.cmi.2017.02.024.

12. Requena-Méndez A, Buonfrate D, Gomez-Junyent J, Zammarchi L, Bisoffi Z, Muñoz J. Evidence-based guidelines for screening and management of strongyloidiasis in non-endemic countries. Am J Trop Med Hyg. 2017;97:645-52. https://doi.org/10.4269/ ajtmh.16-0923.

13. Stauffer WM, Alpern JD, Walker PF. "COVID-19 and dexamethasone: a potential strategy to avoid steroid-related strongyloides hyperinfection. JAMA. 2020. https://doi.org/10.1001/jama.2020. 13170.

14. Clemente WT, et al. Recommendations for management of endemic diseases and travel medicine in solid-organ transplant recipients and donors: Latin America. Transplantation. 2018;102:193-208. https://doi.org/10.1097/TP.0000000000 002027.

15. Calleri G, Angheben A, Albonico M. Neglected tropical diseases in Europe: rare diseases and orphan drugs? Infection. 2019;47:35. https://doi.org/10.1007/s15010-018-1241-2.

16. Tosti ME, Marceca M, Eugeni E, D’Angelo F, Geraci S, Declich S, Della Seta M, Ferrigno L, Marrone R, Pajno C, Pizzarelli S, Rosso A, De Ponte G, Mirisola C, Baglio G. Health assessment for migrants and asylum seekers upon arrival and while hosted in reception centres: Italian guidelines. Health Policy. 2021;125(3):393-405. PMID: 33461797. https://doi.org/10.1016/j. healthpol.2020.12.010

17. Tilli M, Olliaro P, Gobbi F, Bisoffi Z, Bartoloni A, Zammarchi L. Neglected tropical diseases in non-endemic countries in the era of COVID-19 pandemic: the great forgotten. J Travel Med. 2020. https://doi.org/10.1093/jtm/taaa179. 